\title{
A reduced nonlinear model for the simulation of two phase flow in a horizontal pipe
}

Matteo Pischiutta, Gianni Arioli and Alberto Di Lullo

\begin{abstract}
In the last 10 years many 3D numerical schemes have been developed for the study the flow of a mixture of liquid and gas in a pipeline $[2,3,5,9]$, but although they offer a very good accuracy, they are rarely fit for modeling a long pipe, due to the high computational costs. Then one is usually led to consider 1D models, see e.g. the works of Issa and his group [6]. Such models offer much faster simulations than 3D schemes, on the other hand they almost completely miss the dynamics in the transversal direction. Here we present a model able of representing the full 3D dynamics, but with the computational cost typical of 1D simulation. The main feature of our model consists in describing the dynamical variables in the direction transversal to the pipe by means of a family of functions depending on a set of parameters. The model is then solved by a standard finite volume scheme.
\end{abstract}

\section{Introduction}

A common starting point for the simulation of the flow of a two phase fluid in a pipeline is provided by the Reynolds averaged Navier-Stokes equations (RANS) [4,8], which for the $k$-th phase read:

$$
\begin{aligned}
& \frac{\partial}{\partial t}\left(\rho_{k} \alpha_{k}\right)+\nabla \cdot\left(\rho_{k} \alpha_{k} \mathbf{U}_{k}\right)=0, \\
& \frac{\partial}{\partial t}\left(\rho_{k} \alpha_{k} \mathbf{U}_{k}\right)+\nabla \cdot\left(\rho_{k} \alpha_{k} \mathbf{U}_{k} \otimes \mathbf{U}_{k}\right)+\nabla \cdot\left(\alpha_{k} \mathbf{R}_{k}^{e f f}\right)=-\alpha_{k} \nabla p_{k}+\rho_{k} \alpha_{k} \mathbf{g}+\mathbf{M}_{k},
\end{aligned}
$$

Matteo Pischiutta and Gianni Arioli

MOX, Department of Mathematics, Politecnico di Milano

e-mail: matteo.pischiutta,gianni.arioli@polimi.it

Alberto Di Lullo

ENI S.p.A, Exploration and Production Division, e-mail: alberto.dilullo@eni.com 
where $k$ denotes the phase $(k=g, l), \alpha_{k}$ is the fraction of volume occupied by the phase such that $\alpha_{g}+\alpha_{l}=1, \mathbf{U}_{k}$ is the velocity, $p_{k}$ is the pressure, $\mathbf{R}_{k}^{\text {eff }}=$ $-\rho_{k} v_{k}^{\text {eff }}\left(\nabla \mathbf{U}_{k}+\left(\nabla \mathbf{U}_{k}\right)^{T}\right)$ is the strain tensor, which takes into account both viscous and turbolent effects, $v_{k}^{\text {eff }}=v_{k}+v_{t, k}$ is the effective viscosity, and $\mathbf{M}_{k}$ describes the exchange of momentum between the phases.

Our main purpose is to build a fast numerical scheme that can simulate a full 3D flow.

\section{Equation reduction}

We partition the length of the pipeline $x=[0, L]$ in $N$ uniform cells and we set $x_{i}, i=$ $1, \ldots, N$ to be the centers of the cells. Consider a cell $\mathscr{V}$, that is a cylinder bounded by the disks $\mathscr{A}_{\text {in }}, \mathscr{A}_{\text {out }}$ and the wall $\mathscr{W}$. Assuming constant densities, integrating equations (1)-(2) in $\mathscr{V}$ and using Gauss' theorem we get:

$$
\begin{aligned}
& \frac{\partial}{\partial t} \int_{\mathscr{V}} \rho_{k} \alpha_{k}+\int_{\partial \mathscr{V}} \rho_{k} \alpha_{k} \mathbf{U}_{\mathbf{k}} \cdot \mathbf{n}=0, \\
& \frac{\partial}{\partial t} \int_{\mathscr{V}} \rho_{k} \alpha_{k} \mathbf{U}_{k}+\int_{\partial \mathscr{V}} \rho_{k} \alpha_{k}\left(\mathbf{U}_{k} \otimes \mathbf{U}_{k}\right) \cdot \mathbf{n}+\int_{\partial \mathscr{V}}\left(\alpha_{k} \mathbf{R}_{k}^{e f f}\right) \cdot \mathbf{n}=-\int_{\mathscr{V}} \alpha_{k} \nabla p_{k}+\int_{\mathscr{V}} \rho_{k} \alpha_{k} \mathbf{g}+\int_{\mathscr{V}} \mathbf{M}_{k},
\end{aligned}
$$

where $\partial \mathscr{V}=\mathscr{A}_{\text {in }} \cup \mathscr{A}_{\text {out }} \cup \mathscr{W}$ e $\mathbf{n}$ is the normal vector to the surface.

We assume that the transversal components of the velocity are zero, $\mathbf{U}_{k}=\left(u_{k}, 0,0\right)^{T}$, where $u_{k}=u_{k}(x, y, z)$, and constant densities. Then $\mathbf{M}_{k}=\left(M_{k}, 0,0\right)$. We enforce the no-slip boundary condition on the wall, $\left.u_{k}\right|_{\mathscr{W}}=0$, so that the conservation equations become:

$$
\begin{aligned}
& \frac{\partial}{\partial t} \int_{\mathscr{V}} \alpha_{k}+\left[\int_{\mathscr{A}} \alpha_{k} u_{k}\right]_{\text {in }}^{\text {out }}=0, \\
& \frac{\partial}{\partial t} \int_{\mathscr{V}} \alpha_{k} u_{k}+\left[\int_{\mathscr{A}} \alpha_{k} u_{k}^{2}\right]_{\text {in }}^{\text {out }}-\left[\int_{\mathscr{A}} 2 \alpha_{k} v_{k}^{\text {eff }} \frac{\partial u_{k}}{\partial x}\right]_{\text {in }}^{\text {out }}=-\int_{\mathscr{V}} \frac{\alpha_{k}}{\rho_{k}} \frac{\partial p_{k}}{\partial x}+\int_{\mathscr{W}} \alpha_{k} v_{k}^{\text {eff }} \frac{\partial u_{k}}{\partial \mathbf{n}}+\int_{\mathscr{V}} \frac{M_{k}}{\rho_{k}}
\end{aligned}
$$

where $\left[\int_{\mathscr{A}} \psi\right]_{\text {in }}^{\text {out }}=\int_{\mathscr{A}_{\text {out }}} \psi-\int_{\mathscr{A}_{\text {in }}} \psi$.

Our model consists in describing the transverse profile of the unknown variables $\alpha_{k}$ and $u_{k}$ at each point in space through given functions depending on some parameters which in turn vary in the longitudinal direction. Our choice for $\alpha_{g}(x, y, z)$ is as follows:

$$
\alpha_{g}(x, y, z)= \begin{cases}0 & y \leq \beta(x), \\ \frac{y-\beta(x)}{\delta(x)} & \beta(x)<y<\beta(x)+\delta(x), \\ 1 & y \geq \beta(x)+\delta(x),\end{cases}
$$

where $\beta(x)$ is related to the level of the liquid and $\delta(x)$ is the width of the interface. Concerning the velocity profiles we choose a parabolic profile in $z$ : 


$$
u_{k}(x, y, z)=U_{k}(x, y) \frac{R^{2}-y^{2}-z^{2}}{R^{2}-y^{2}},
$$

where $R$ is the constant radius of the pipe, then we choose a linear dependence on $y$ for the liquid phase:

$$
U_{l}(x, y)=\frac{y+R}{\beta(x)+R} \gamma(x), \quad y \leq \beta(x)+\delta(x),
$$

and quadratic for the gas phase:

$$
U_{g}(x, y)=\zeta(x)(y-R)(\beta(x)-y)+\gamma(x) \frac{y-R}{\beta(x)-R}, \quad y \geq \beta(x) .
$$

The graphs of the center line profiles of the variables are reported in Fig.1.
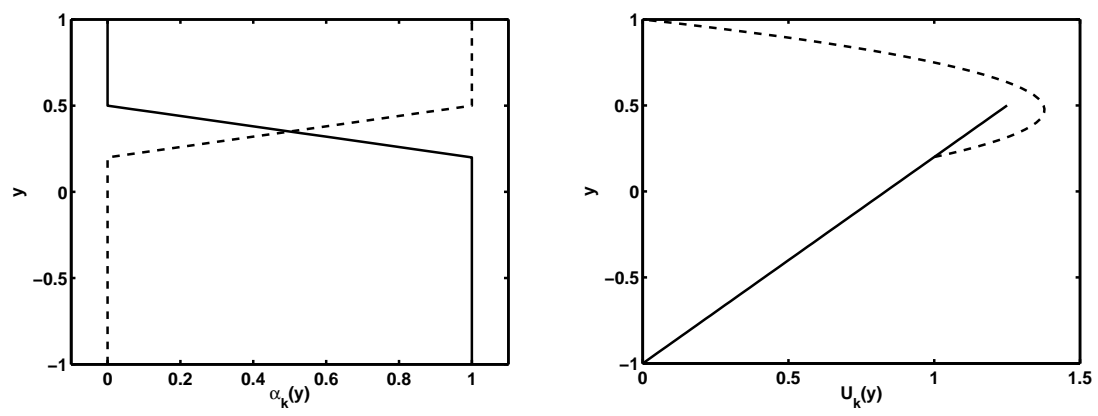

Fig. 1 Left: graph of $\alpha_{k}$, right: graph of $U_{k}$. In solid line, the graphs relative to the liquid phase, in broken line, the graphs relative to the gas phase. The parameter are: $\beta=0.2, \delta=0.3, \gamma=1$, $\zeta=5$.

We assume that the pressure of the gas is constant in the transversal directions, while the liquid is subject to the hydrostatic pressure:

$$
p_{g}(x, y, z)=P(x), \quad p_{l}(x, y, z)=P(x)+\rho_{l} g(\beta+\delta / 2-y),
$$

which leads to a term proportional to the slope of the free surface $\partial(\beta+\delta / 2) / \partial x$ in the momentum equation of the liquid phase. We adopt a zero-equation model for the computation of the turbolent viscosity [1], that is $v_{k}=\frac{\bar{U}_{k} l_{k}}{R e_{k}}$, where $\bar{U}_{k}$ is the maximum phase velocity, $l_{k}$ is a geometric length scale and $R e_{k}=1000$ is a free parameter. 


\section{Numerical integration}

We assume that all the unknowns are constant in the cell and located in the centers of the cells. Hence, all integrals in (5-6) can be computed explicitly, obtaining functions of the parameters $\beta, \delta, \gamma$ e $\zeta$. We set the following notation:

$$
\begin{aligned}
& \mathbb{H}_{l}(\beta, \delta):=\int_{\mathscr{A}} \alpha_{l}, \quad \mathbb{H}_{g}(\beta, \delta):=\int_{\mathscr{A}} \alpha_{g}, \\
& \gamma \mathbb{F}_{l}(\beta, \delta):=\int_{\mathscr{A}} \alpha_{l} u_{l}, \quad \zeta \mathbb{F}_{g, 1}(\beta, \delta)+\gamma \mathbb{F}_{g, 2}(\beta, \delta):=\int_{\mathscr{A}} \alpha_{g} u_{g}, \\
& \mathbb{G}_{l}(\beta, \delta, \gamma):=\int_{\mathscr{A}} \alpha_{l} u_{l}^{2}, \quad \mathbb{G}_{g}(\beta, \delta, \gamma, \zeta):=\int_{\mathscr{A}} \alpha_{l} u_{g}^{2}, \\
& \mathbb{W}_{l}(\beta, \delta, \gamma):=\int_{\mathscr{W}} \alpha_{l} \frac{\partial u_{l}}{\partial \mathbf{n}}, \quad \mathbb{W}_{g}(\beta, \delta, \gamma, \zeta):=\int_{\mathscr{W}} \alpha_{g} \frac{\partial u_{g}}{\partial \mathbf{n}}
\end{aligned}
$$

Time is discretized with explicit Euler method. Equation (5) reads (subscripts denote the cells, superscripts denote time):

$$
\int_{\mathscr{V}} \alpha_{i}^{n+1}=\int_{\mathscr{V}} \alpha_{i}^{n}+\Delta t\left[\int_{\mathscr{A}} \alpha_{i-1 / 2}^{n} u_{i-1 / 2}^{n}-\int_{\mathscr{A}} \alpha_{i+1 / 2}^{n} u_{i+1 / 2}^{n}\right] .
$$

The width of the interface should be dictated by some closure equation, $\delta_{i}^{n+1}=$ $\mathbb{B}\left(\beta_{i}^{n}, \delta_{i}^{n}, \gamma_{i}^{n}, \zeta_{i}^{n}\right)$, but our current choice is to keep it constant. We found an optimal value at $\delta=10^{-2} \mathrm{~mm}$. We remark that the width of the interface affects the drag between the phases.

We use the mass conservation equation of the liquid phase to update the free surface level $\beta$ :

$$
\mathbb{H}_{l}\left(\beta_{i}^{n+1}, \delta_{i}^{n+1}\right)=\mathbb{H}_{l}\left(\beta_{i}^{n}, \delta_{i}^{n}\right)+\frac{\Delta t}{\Delta x}\left[\gamma_{i-1 / 2}^{n} \mathbb{F}_{l}\left(\beta_{i-1 / 2}^{n}, \delta_{i-1 / 2}^{n}\right)-\gamma_{i+1 / 2}^{n} \mathbb{F}_{l}\left(\beta_{i+1 / 2}^{n}, \delta_{i+1 / 2}^{n}\right)\right],
$$

that is a nonlinear equation in $\beta_{i}^{n+1}$ which is solved in every cell with standard Newton method. The conservation of the gas phase is used to obtain an equation for the pressure. To compute the interface values $\gamma_{i-1 / 2}^{n}, \beta_{i-1 / 2}^{n}$ and $\delta_{i-1 / 2}^{n}$ we have to interpolate. Since we are considering only positive velocities we chose the upwind interpolation (e.g.: $\left.\beta_{i-1 / 2}^{n}=\beta_{i-1}^{n}\right)$.

We discretize the equation for the conservation of momentum for the liquid phase as follows:

$$
\begin{aligned}
\frac{\partial}{\partial t} \int_{\mathscr{V}} \alpha_{l} u_{l} & \simeq \Delta x \frac{\partial}{\partial t}\left(\gamma_{i} \mathbb{F}_{l}\left(\beta_{i}, \delta_{i}\right)\right) \simeq \Delta x \frac{\Delta\left(\gamma_{i} \mathbb{F}_{l}\left(\beta_{i}, \delta_{i}\right)\right)}{\Delta t} \\
{\left[\int_{\mathscr{A}} \alpha_{l} u_{l}^{2}\right]_{\text {in }}^{\text {out }} } & \simeq \mathbb{G}_{l}\left(\beta_{i+1 / 2}, \delta_{i+1 / 2}, \gamma_{i+1 / 2}\right)-\mathbb{G}_{l}\left(\beta_{i-1 / 2}, \delta_{i-1 / 2}, \gamma_{i-1 / 2}\right), \\
-\int_{\mathscr{V}} \frac{\alpha_{l}}{\rho_{l}} \frac{\partial p_{l}}{\partial x} & =-\int_{\mathscr{V}} \frac{\alpha_{l}}{\rho_{l}} \frac{\partial P}{\partial x}-\int_{\mathscr{V}} \alpha_{l} g \frac{\partial(\beta+\delta / 2)}{\partial x} \simeq-\Delta x \mathbb{H}_{l}\left(\beta_{i}, \delta_{i}\right)\left(\left.\frac{1}{\rho_{l}} \frac{\partial P}{\partial x}\right|_{i}+\left.g \frac{\partial(\beta+\delta / 2)}{\partial x}\right|_{i}\right) .
\end{aligned}
$$


The gradient of the pressure will be discussed later, while the slope of the free surface is handled with a centered scheme. Since the viscosity is constant in the section of the tube, we have:

$$
\int_{\mathscr{W}} \alpha_{l} v_{l}^{e f f} \frac{\partial u_{l}}{\partial \mathbf{n}} \simeq v_{l, i}^{e f f} \mathbb{W}_{l}\left(\beta_{i}, \delta_{i}, \gamma_{i}\right)
$$

The drag force between the phases is computed with $[4,8]$ :

$$
M_{l}=\frac{3}{4} \alpha_{l} \alpha_{g}\left(\alpha_{l} \frac{C_{D, l} \rho_{g}}{d_{l}}+\alpha_{g} \frac{C_{D, g} \rho_{l}}{d_{g}}\right)\left|u_{r}\right| u_{r},
$$

where $d_{l}$ and $d_{g}$ are the typical diameters of the drops/bubbles of the phases, $u_{r}=$ $u_{g}-u_{l}$ is the relative velocity and $C_{D, l}, C_{D, g}$ are coefficients computed with SchillerNaumann formula. Since $\alpha_{l} \alpha_{g} \neq 0$ only at the interface $\beta<y<\beta+\delta$, we have:

$$
\int_{\mathscr{V}} \frac{M_{l}}{\rho_{l}} \simeq \Delta x \int_{\beta}^{\beta+\delta}\left(\int_{-\sqrt{R^{2}-y^{2}}}^{-\sqrt{R^{2}-y^{2}}} \frac{M_{l}}{\rho_{l}} d z\right) d y=\frac{\Delta x}{\rho_{l}} \mathbb{M}_{l}\left(\beta_{i}, \delta_{i}, \gamma_{i}, \zeta_{i}\right) .
$$

The integrals are computed with a quadrature scheme. We compute the diffusion term using the average velocities:

$$
\bar{u}_{l}=\frac{\int_{\mathscr{A}} \alpha_{l} u_{l}}{\int_{\mathscr{A}} \alpha_{l}}=\gamma \frac{\mathbb{F}_{l}(\beta, \delta)}{\mathbb{H}_{l}(\beta, \delta)}
$$

approximating $\partial u_{l} / \partial x$ with $\partial \bar{u}_{l} / \partial x$ (i.e. a term that does not depend on $y$ and $z$ ). Then we define:

$$
\begin{aligned}
{\left[\int_{\mathscr{A}} 2 \alpha_{l} v_{l}^{\text {eff }} \frac{\partial \bar{u}_{l}}{\partial x}\right]_{\text {in }}^{\text {out }} } & \simeq\left[2 \frac{\partial \bar{u}_{l}}{\partial x} v_{l}^{\text {eff }} \int_{\mathscr{A}} \alpha_{l}\right]_{i n}^{\text {out }} \\
& \left.:=\left[\mathbb{D}_{l}\left(\beta_{i+1 / 2}, \delta_{i+1 / 2}, \gamma_{i+1 / 2}\right)-\mathbb{D}_{l}\left(\beta_{i-1 / 2}, \delta_{i-1 / 2}, \gamma_{i-1 / 2}\right)\right)\right] .
\end{aligned}
$$

Finally, the momentum equation for the liquid phase reads:

$$
\begin{aligned}
\Delta x \frac{\Delta\left(\gamma_{i} \mathbb{F}_{l}\left(\beta_{i}, \delta_{i}\right)\right)}{\Delta t}+ & {\left.\left[\mathbb{G}_{l}\left(\beta_{i+1 / 2}, \delta_{i+1 / 2}, \gamma_{i+1 / 2}\right)-\mathbb{G}_{l}\left(\beta_{i-1 / 2}, \delta_{i-1 / 2}, \gamma_{i-1 / 2}\right)\right)\right] } \\
& \left.-\left[\mathbb{D}_{l}\left(\beta_{i+1 / 2}, \delta_{i+1 / 2}, \gamma_{i+1 / 2}\right)-\mathbb{D}_{l}\left(\beta_{i-1 / 2}, \delta_{i-1 / 2}, \gamma_{i-1 / 2}\right)\right)\right]= \\
& -\Delta x \mathbb{H}_{l}\left(\beta_{i}, \delta_{i}\right)\left(\left.\frac{1}{\rho_{l}} \frac{\partial P}{\partial x}\right|_{i}+\left.g \frac{\partial(\beta+\delta / 2)}{\partial x}\right|_{i}\right) \\
& +v_{l, i}^{e f f} \mathbb{W}_{l}\left(\beta_{i}, \delta_{i}, \gamma_{i}\right)+\frac{\Delta x}{\rho_{l}} \mathbb{M}_{l}\left(\beta_{i}, \delta_{i}, \gamma_{i}, \zeta_{i}\right),
\end{aligned}
$$

which is a linear equation for the unknown $\gamma_{i}^{n+1}$. The gas phase is handled similarly, leading to a linear equation for the unknown $\zeta_{i}^{n+1}$.

The equations are integrated according to the following scheme:

1. Mass conservation: The free surface is updated solving (17) for each cell. 
2. Velocity predictor: The auxiliar values $\gamma^{*}$ and $\zeta^{*}$ are computed with an explicit Euler iteration of the momentum equations.

3. Pressure correction: to compute the pressure correction $\left.\frac{\partial P}{\partial x}\right|_{i} ^{*}$ we enforce the continuity equation.

4. Velocity correction: Once $\left.\frac{\partial P}{\partial x}\right|_{i} ^{*}$ is known, we can compute $\gamma^{n+1}$ e $\zeta^{n+1}$.

\section{Results}

Figure 2 represents some results of our simulations. The picture on the left displays some snapshots of the free surface in slug flow at different instants in time; the curves are represented each $0.2 \mathrm{sec}$ and are shifted relative to each other with respect to time. The liquid superficial velocity is $L S V=0.7 \mathrm{~m} / \mathrm{s}$ and the gas superficial velocity is $G S V=6 \mathrm{~m} / \mathrm{s}$, in a pipeline with $4 \mathrm{~cm}$ of diameter and $10 \mathrm{~m}$ long. We can clearly observe that our model can reproduce the continuous generation of slug in the pipe.

The picture on the right compares our results for different regimes to the benchmark given by the flow map provided in [7]. We observe that our model is able to reproduce a good transitions between the elongated bubble and the slug regimes, whereas the exact transition between the stratified/wave flow and the slug flow is more difficult to be captured. Moreover, we cannot distinguish the stratified from the wave regime. Nevertheless, the comparison with the experimental data show that our model is able to predict the flow map with an acceptable accuracy.
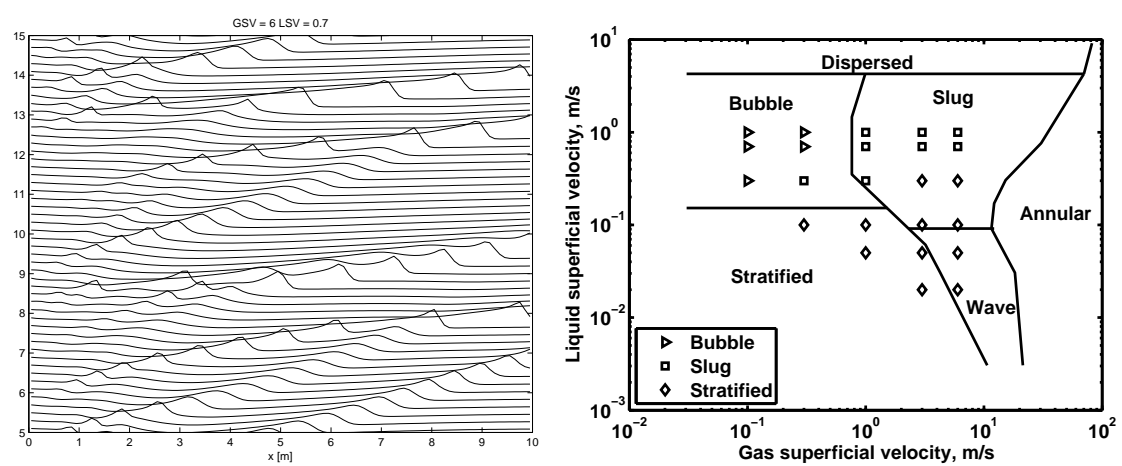

Fig. 2 Shapshots (left) and flow map (right). 


\section{Conclusions}

These are the main features of the scheme that we introduced:

- The numerical algorithm is very fast, it is comparable with $1 \mathrm{~d}$ schemes.

- The results are in quantitative and qualitative accord with experimental data.

- The model is very flexible. The transversal profiles can be changed, while keeping the basic structure of the model and its numerical implementation.

- The numerical algorithm is stable, it does not pose any problem in the slug regions.

- The model has been implemented for horizontal pipelines. It could be easily adapted to moderate slopes, but it requires serious modifications to handle vertical pipelines.

In conclusion, we proved the feasibility of a reduction scheme for two phase fluidodynamics in a pipeline, consisting in choosing a specific shape for the dynamic variables in the transversal directions depending on parameters.

\section{Acknowledgment}

The financial support from ENI S.p.A. is gratefully acknowledged.

\section{References}

1. Ansys, CFX: Ansys CFX-Solver Theory Guide (2010)

2. Bartosiewicz, Y., Seynhaeve, J.M., Vallée, C., Höhne, T., Laviéville, J.M.: Modeling free surface flows relevant to a PTS scenario: Comparison between experimental data and three RANS based CFD-codes. Comments on the CFD-experiment integration and best practice guideline. Nuclear Engineering and Design 240(9), 2375-2381 (2010)

3. Frank, T.: Numerical simulation of slug flow regime for an air-water two-phase flow in horizontal pipes. In: The 11th International Topical Meeting on Nuclear Reactor Thermal-Hydraulics (NURETH-11). Avignon, France (2005)

4. Hill, D.P.: The computer simulation of dispersed two-phase flow. Ph.D. thesis, University of London (1998)

5. Höhne, T.: Experiments and numerical simulations of horizontal two-phase flow regimes. In: Proceeding of the Seventh International Conference on CFD in the Minerals and Process Industries, Melbourne, Australia (2009)

6. Issa, R., Kempf, M.: Simulation of slug flow in horizontal and nearly horizontal pipes with the two-fluid model. International Journal of Multiphase Flow 29(1), 69-95 (2003)

7. Mandhane, J., Gregory, G., Aziz, K.: A flow pattern map for gasliquid flow in horizontal pipes. International Journal of Multiphase Flow 1(4), 537 - 553 (1974)

8. Rusche, H.: Computational fluid dynamics of dispersed Two-Phase flows at high phase fractions. Ph.D. thesis, Imperial College (2002)

9. Vallée, C., Höhne, T., Prasser, H.M., Sühnel, T.: Experimental investigation and CFD simulation of horizontal stratified two-phase flow phenomena. Nuclear Engineering and Design 238(3), 637-646 (2008) 